\title{
CÓMO FUNCIONAN LAS COMPETENCIAS SOCIOEMOCIONALES EN LOS ESTUDIANTES DE ALTA HABILIDAD
}

\section{How socio-emotional competences work on students with bigh abilitie}

Marta Sainz Gómez, Rosario Bermejo García, Carmen Ferrándiz García, M. a Dolores Prieto SÁncheZ y María José Ruiz Melero

Universidad de Murcia

Correo-e: m.gomez@um.es; charo@um.es; carmenfg@um.es; lola@um.es; mariajose.ruiz4@um.es

Recepción: 17 de noviembre de 2014

Envío a informantes: 3 de diciembre de 2014

Aceptación definitiva: 13 de enero de 2015

Biblid. [0214-3402 (2015) (II época) n. ${ }^{\circ}$ 2I; 33-47]

RESUMEN: El objetivo del trabajo consiste en estudiar las competencias socioemocionales en un grupo de talentos. En el estudio han participado I237 estudiantes de Educación Secundaria Obligatoria (ESO); de los cuales 23 I alumnos, un 8,3\%, han sido identificados como talentos figurativos; un 2,7\% como talentos académicos, y un $7,6 \%$ como talentos combinados (figurativos y académicos), de los cuales I45 son chicos (el 62,8\%). El rango de edad está comprendido entre los II y los I7 años $(M=$ I3,91; $D T=1,182)$. Todos ellos cursan sus estudios en distintos centros tanto públicos como concertados de España. La identificación se hizo de acuerdo a los criterios de Castelló y Batlle (1998). El instrumento utilizado para evaluar las competencias socioemocionales ha sido el Inventario de Inteligencia Emocional (Emotional Quotient Inventory EQ-i: Youth Version. EQ-i:YV, Bar-On y Parker, 2000). Es un autoinforme que consta de 60 ítems orientado a medir la IE de niños y adolescentes con edades comprendidas entre 7 y i8 años. Los datos señalan que los talentos figurativos manifiestan un alto manejo del estrés; mientras que los talentos combinados (académicos y figurativos) muestran elevadas competencias intrapersonales y de adaptabilidad.

Palabras Clave: talentos académicos; figurativos; competencias socioemocionales.

AвSTRACT: The aim of this paper is to study social-emotional competences of a group of talented students. For this purpose 1237 secondary school students have participated, in which $23 \mathrm{I}$ students a $8.3 \%$ were identified as figurative talents, a $2.7 \%$ as academical 
talents and a $7.6 \%$ as combined talents (figurative and academical), where 145 (62.6\%) were boys. All of them are studying in different education centres in Spain both public and private. Identification was made according to the criteria developed by Castelló $\&$ Battle (1998). The students ages range from in to 17 years old $(M=13.91 ; D T=$ I.I82). The instrument used to measure social-emotional competences was the Emotional Quotient Inventory: Youth Version, EQ-i:Yv (Bar-On \& Parker, 2000). The Bar-On EQ-i:Yv is a 6o-item self-report inventory which assesses emotional intelligence (EI) in youths aged from 7 to 18 years old. The results indicate that figurative talented students show a higher stress management; while the combinated talents (figurative and academical talent) show higher intrapersonal and adaptability competences.

KEY WORDS: academical talents; figurative; social-emotional compentences.

$\mathrm{E}$ S CIERTO QUe La COMPLEJa CONFIGURACión COGNITIVA de los estudiantes con altas habilidades comporta también una cierta complejidad e incluso diversidad de sus aspectos emocionales y de personalidad, porque suelen tener vivencias más intensas y complejas que las de sus compañeros (Gross, 20I4; Peterson, 20I4; Sword, 2005). De hecho, algunos expertos afirman que los superdotados muestran una mayor conciencia, mayor sensibilidad y una mayor capacidad para entender y transformar las percepciones intelectuales y emocionales en experiencias. Las emociones no se pueden tratar por separado de la inteligencia, porque se entrelazan y se influyen mutuamente. Los pensamientos y las emociones de los superdotados difieren de las demás, y como resultado perciben su mundo y reaccionan de otra manera (Kane y Silverman, 20r4; Neville, Tolan y Piechowski, 2013).

Los trabajos pioneros de Terman y sus colaboradores (Burks, Jensen y Terman, I930; Cox, 1926; Terman, I916, I925; Terman y Oden, I947, I959), así como el de Hollingworth (I93I, I942) y otros (Judá, I949; Lewis, I943), indicaron que las personas con capacidad cognitiva muy elevada eran superiores a la media en casi todos los aspectos referidos al ajuste psicológico y bienestar físico y social. En este mismo sentido, son diversos los autores que indican que los superdotados adolescentes desde muy pequeños afrontan las situaciones de estrés mejor, porque tienen un sentido más agudo sobre las diversas posibilidades de afrontamiento al estrés; o, dicho de otra manera, los superdotados están más abiertos y son más flexibles para afrontar los problemas (Dirkes, I983; Schuler, 2002). En este mismo sentido, otros autores demostraron que los superdotados manifestaban menores niveles de ansiedad que los no superdotados (Zeidner y Schleyer, I999). Esta capacidad de afrontamiento al estrés y la flexibilidad se explican por la gran capacidad del superdotado para entenderse a sí mismo y para entender a los demás, esto hace que se enfrenten mejor al estrés y a los conflictos (Baker, 1995; Galluci, I988; Grossberg y Cornell, I988; Howard, Hamilton y Franks, I995; Nail y Evans, I997; Neihart, I99i; OlszewskiKubilius y Kukieke, I989). En definitiva, lo que vienen a demostrar estos trabajos es que los superdotados generalmente interaccionan con la complejidad del ambiente relativamente bien y suelen tener una serie de habilidades para afrontar los problemas y ajustarse positivamente.

Sin embargo, existe otra postura, mantenida por diversos investigadores, que sostiene que los estudiantes de altas habilidades (superdotados y talentos) tienen un mayor riesgo de desarrollar problemas de ajuste psicológico, con respecto a sus iguales, 
y que la superdotación incrementa la vulnerabilidad del niño hacia las dificultades de ajuste psicológico (Austin y Draper, I98I; Janos y Robinson, I985; Lajoie y Shore, I98I; Powell y Haden, I984; Roedell, I984; Schauer, 1976). Quizás, es su perfeccionismo asociado a la superdotación lo que les genera ciertos niveles de ansiedad y, por tanto, desajuste emocional (Pyryt, 20II).

Desde que aparecen los nuevos modelos de la Inteligencia Emocional, se han realizado diferentes trabajos orientados a evaluar los componentes y habilidades de la misma, estudiando cómo dichos componentes funcionan en los estudiantes con alta habilidad. Analizaremos principalmente los trabajos realizados con el modelo de BarOn (1997), por ser el que hemos utilizado en nuestro trabajo empírico.

Si nos centramos en la variable manejo del estrés (capacidad para tolerar el estrés, controlar los impulsos y trabajar bien bajo presión sin desmoronarse) y la alta habilidad (superdotados y talentos), los resultados son diferentes. Por ejemplo, hay autores cuyos estudios indicaron que los estudiantes superdotados manifiestan un adecuado control del estrés (Schwean, Saklofske, Widdifield-Konkin, Parker y Kloosterman, 2006). Mientras, otros señalaron que los superdotados puntuaron por debajo de las puntuaciones estándar en la dimensión manejo del estrés. Estos datos sugieren que los superdotados eran más propensos al enfado y mostraban mayor dificultad para afrontar las situaciones estresantes (Seon-Young y Olszewski-Kubilius, 2006). Los resultados de Ferrando (2006) mostraron que los alumnos con alta habilidad obtuvieron menor puntuación en la dimensión manejo del estrés que los estudiantes de habilidades medias; mientras que los talentos manifestaron mayor manejo del estrés que los superdotados (Prieto y Ferrando, 2009).

Bar-On analizó la relación entre la IE y la superdotación. Pretendió estudiar la relación entre la IE y la inteligencia psicométrica, el rendimiento académico, el nivel de la autoactualización y la capacidad para resolver los problemas (Bar-On, 2007; Bar-On y Maree, 2009). Los datos sobre la relación entre la IE y la inteligencia psicométrica demostraron que el manejo del estrés era la dimensión que mejor predecía la inteligencia psicométrica de los alumnos, sugiriendo que ésta estaba fuertemente influenciada por la capacidad para manejar las emociones, más que las otras dimensiones (asertividad, empatía, capacidad para resolver problemas, optimismo o la validación de los propios sentimientos y pensamientos) (Bar-On, 2004, 2007; Bar-On y Maree, 2009). Respecto a la relación entre la IE y el rendimiento académico, se concluyó que el manejo del estrés era uno de los predictores más fuertes del rendimiento académico, aunque también otras dimensiones como la validación de los sentimientos y del pensamiento, la resolución de problemas, la autoactualización y el optimismo. Respecto a la relación entre la IE y la autoactualización, los resultados no mostraron relación alguna entre el manejo del estrés y la autoactualización. Por último, cuando se relacionaron la IE y la capacidad para resolver problemas, se encontró que el manejo del estrés fue el mejor predictor de la destreza para la resolución de problemas (Bar-On, 200I, 2007; Bar-On y Maree, 2009).

Sainz (2008) indicó que los superdotados y los talentos se autovaloraron con una buena capacidad para manejar las situaciones estresantes, mejor que sus compañeros de aula. Más tarde, Sainz (20IO) estudió la IE y su relación con la inteligencia psicométrica (alta, media y baja). Los datos indicaron que los estudiantes con alta inteligencia tenían un adecuado manejo del estrés; sin embargo, sus compañeros con baja inteligencia manifestaron unas ciertas carencias en el manejo del estrés. También 
Fernández-Vidal (20II) halló que los alumnos con alta habilidad tienen ligeramente un mayor manejo del estrés que los alumnos sin alta habilidad.

Son también diversos los estudios sobre la adaptabilidad (capacidad para gestionar el cambio, evaluar correctamente la realidad, ser flexible ante nuevas situaciones, así como para crear soluciones y resolver problemas) y la alta habilidad (superdotados y talentos). La razón fundamental de estos primeros estudios consistió en descartar el estereotipo referido a la inestabilidad de los alumnos con alta inteligencia que viene manteniéndose desde los primeros estudios de la superdotación (Janos y Robinson, 1985; Powell y Haden, 1984). Por ejemplo, Schwean et al. (2006) indicaron que los superdotados que asisten al aula ordinaria manifiestan mayor adaptabilidad que los que asisten a programas específicos para superdotados. Los padres y profesores también informaron de la superioridad en la adaptabilidad de sus hijos y alumnos (superdotados que asisten al aula ordinaria), respectivamente. En este mismo sentido, en otro estudio se destacó que los superdotados puntuaron por encima del promedio del baremo en la dimensión adaptabilidad en las escalas de BarOn, lo que indica que se percibían con gran capacidad para tratar con los problemas cotidianos (Seon-Young y Olszewski-Kubilius, 2006). Ferrando (2006) demostró que los alumnos con altas habilidades (superdotados y talentos) obtuvieron puntuaciones más elevadas que sus compañeros en todas las áreas de la IE, siendo estas diferencias más significativas para la adaptabilidad. Los superdotados manifestaban mayor flexibilidad y destrezas para ajustar sus emociones, pensamientos y comportamiento al cambiar de situaciones, así como una mayor habilidad para definir, identificar e implementar soluciones a los problemas.

Los estudios de Bar-On y sus colegas informan de una relación entre adaptabilidad e inteligencia psicométrica. Por tanto, se concluyó que la autoactualización y la adaptabilidad son un buen predictor de la misma. Por último, el autor encontró que la adaptabilidad estaba relacionada con una mayor capacidad para resolver los problemas (Bar-On, 200I, 2004, 2007; Bar-On y Maree, 2009).

Sainz (2008) encontró que los superdotados y talentos se autopercibieron con una mayor adaptabilidad que los no superdotados. Más tarde, Sainz (2010) observó que el grupo de estudiantes de alta inteligencia se percibió con mayor adaptabilidad que sus compañeros con baja inteligencia. En esta misma línea, los datos de Fernández-Vidal (20II) indicaron que los alumnos con alta habilidad (superdotados y talentos) tienen mayor adaptabilidad en comparación a los alumnos sin alta habilidad.

Los trabajos sobre estado de ánimo (optimismo, felicidad, funcionamiento social y emocional) y la alta habilidad (superdotados y talentos) muestran resultados interesantes y diferentes. Por ejemplo, hay investigaciones que indicaron la no existencia de diferencias significativas entre alumnos superdotados y de habilidades medias para la variable estado de ánimo (Schwean et al., 2006; Seon-Young y Olszewski-Kubilius, 2006). Mientras que los resultados aportados por Ferrando (2006) mostraron que los superdotados se percibían con mayor estado de ánimo que sus compañeros.

En los trabajos de Bar-On, los resultados mostraron que el estado de ánimo estaba relacionado con la inteligencia psicométrica, con el rendimiento académico y con la capacidad para resolver problemas (Bar-On, 200I, 2007; Bar-On y Maree, 2009). Además, indicaron que es un buen predictor para la autoactualización (Bar-On, 20oI, 2004; Bar-On y Maree, 2009). 
Mientras que Sainz (2008) encontró diferencias marginalmente significativas en el estado de ánimo a favor de los alumnos de alta habilidad. En otro estudio, Sainz (20Io) encontró que los alumnos de alta inteligencia se autopercibieron de forma más favorable que sus compañeros, en la dimensión estado de ánimo. Asimismo, los padres valoraron con mayor estado de ánimo a este grupo de alta inteligencia. Fernández-Vidal (20II) no encontró diferencias estadísticamente significativas en estado de ánimo entre los alumnos con y sin alta habilidad, no obstante, los alumnos con alta habilidad mostraron una tendencia a tener un mejor estado de ánimo que sus compañeros.

Respecto a los trabajos realizados sobre el componente de la inteligencia interpersonal (empatía, responsabilidad social y relaciones sociales) y la alta habilidad, son diferentes los resultados. Por ejemplo, Schwean et al. (2006) indicaron que los no superdotados se autovaloraron con mayor inteligencia interpersonal que los superdotados. Sin embargo, Seon-Young y Olszewski-Kubilius (2006) no hallaron diferencias significativas en las áreas de la inteligencia interpersonal, cuando comparan superdotados con la muestra normativa.

Respecto a los trabajos de Bar-On (2007; Bar-On y Maree, 2009), los resultados indicaron que existe una correlación entre las habilidades interpersonales y la inteligencia psicométrica (Bar-On, 2004, 2007). Con el rendimiento académico no se encontró correlación (Bar-On, 2006; Bar-On y Maree, 2009). En cuanto a la capacidad para resolver problemas, los datos indicaron relación con la dimensión interpersonal (Bar-On, 2007; Bar-On y Maree, 2009) y, por último, dicen los autores que la dimensión interpersonal es un buen predictor de la autoactualización (Bar-On, 200I; Bar-On y Maree, 2009).

Sainz $(2008,2010)$ corroboró que los alumnos con alta habilidad se autoperciben con mayor competencia interpersonal que sus compañeros, aunque, cuando se comparan grupos según su nivel intelectual, las diferencias, según la autora, no eran estadísticamente significativas.

Cuando se analizan los estudios hechos sobre la inteligencia intrapersonal (autoconocimiento emocional, asertividad, autoestima, autodesarrollo e independencia emocional) y la alta habilidad, los resultados indicaron algunas diferencias. Los datos de Schwean et al. (2006) demostraron que los superdotados también se autopercibieron con mayor inteligencia intrapersonal que los no superdotados. Sin embargo, otros investigadores no hallaron diferencias estadísticamente significativas entre la inteligencia intrapersonal de los superdotados y la muestra normativa del inventario de Bar-On (Seon-Young y Olszewski-Kubilius, 2006). En el estudio de Ferrando (2006) los datos revelaron que los superdotados obtuvieron puntuaciones más elevadas en todas las dimensiones de la IE, encontrándose las diferencias más acusadas en la variable intrapersonal. Dice la autora que los superdotados se perciben con mayores habilidades intrapersonales que los talentos.

Bar-On (2007) estableció que existe una relación entre la dimensión intrapersonal, la inteligencia psicométrica, el rendimiento académico, la autoactualización y la capacidad para resolver problemas (Bar-On y Maree, 2009).

En el trabajo realizado por Sainz (2008), los datos mostraron que los superdotados y los talentos se valoran con adecuadas competencias intrapersonales. Además, en este estudio la autora encontró un efecto significativo de la variable excepcionalidad (presencia o no de superdotación) en la dimensión intrapersonal, a favor de los alumnos 
con habilidades medias. Más tarde, Sainz (2010) no encontró diferencias estadísticamente significativas entre los alumnos de alta, media y baja inteligencia para la dimensión intrapersonal valorada por dichos alumnos. Por último, Fernández-Vidal (20II) observó que la inteligencia intrapersonal era más elevada en alumnos sin alta habilidad que en los de alta habilidad (superdotados y talentos) (Sainz, FernándezVidal y Soto, 20I2).

Después de esta revisión, nuestro objetivo se centra en estudiar las competencias socioemocionales de una muestra de estudiantes con talento académico (estudiantes con un alto razonamiento verbal, lógico y gestión de memoria), figurativo (estudiantes con alto razonamiento espacial, lógico y/o mecánico) y combinados (son estudiantes que manifiestan altas puntuaciones en el razonamiento verbal, lógico, espacial, memoria y mecánico). En la Tabla i se muestra con mayor detalle las características de estos talentos.

\section{Método}

\section{Participantes}

En el estudio han participado un total de 1237 estudiantes de Educación Secundaria Obligatoria (ESO); de los cuales 23 I alumnos, un $8,3 \%$, han sido identificados como talentos figurativos; un $2,7 \%$ como talentos académicos, y un 7,6\% como talentos combinados (figurativos más académicos), de los cuales i 45 son chicos (el 62,8\%). La distribución por cursos fue: de primero de la EsO 96 (41,6\%), de segundo is $(6,5 \%)$, de tercero $97(42 \%)$, de cuarto $22(9,5 \%)$ y r $(0,4 \%)$ de r. ${ }^{\circ}$ de Bachillerato. En cuanto al rango de edad de los participantes de la muestra total está comprendido entre los ir y los I7 años $(M=13,91 ; D T=1,182)$. Todos ellos cursan sus estudios en distintos centros tanto públicos como concertados de España. La identificación se hizo de acuerdo a los criterios de Castelló y Batlle (1998) (ver Tabla i).

\section{Instrumento}

Para evaluar las competencias socioemocionales se utilizó el EQ:i-Yv (Bar-On y Parker, 2000) que es un instrumento destinado a la autoevaluación de competencias socioemocionales de niños y adolescentes de 6 a i 8 años. Está compuesto por 60 ítems que se presentan en una escala tipo Likert de cuatro puntos ( $\mathrm{I}=$ Nunca me pasa, 4 = Siempre me pasa) y que evalúan cinco factores: a) intrapersonal o habilidad para comprender las propias emociones y su comunicación a los otros; b) interpersonal o habilidad para entender y apreciar las emociones de los otros; c) adaptabilidad o flexibilidad y eficacia para resolver conflictos; d) manejo del estrés o habilidad para dirigir y controlar las propias emociones; y e) estado de ánimo o habilidad para tener una actitud positiva ante la vida. Además, ofrece una puntuación total o Cociente Emocional de la IE (EQ: Emotional Quotient), resultado de la combinación de los factores anteriores. La fiabilidad global de la prueba obtenida por los autores es de o,88I y para cada uno de los factores el coeficiente alfa de Cronbach es: estado de ánimo $\alpha$ $=0,832$; adaptabilidad $\alpha=0,759$; manejo del estrés $\alpha=0,773$; interpersonal $\alpha=0,692$; intrapersonal $\alpha=0,687$ (Sainz, Ferrándiz, Fernández-Vidal y Ferrando, 20I4). 


\section{Procedimiento}

Para el desempeño de este trabajo se ha contado con la colaboración de los directores de los centros, los profesores de aula, los orientadores y los estudiantes de Educación Secundaria Obligatoria. En primer lugar, se envió una circular a los centros que participaron en el estudio en el que se informaba del inicio del proceso de evaluación, y se informó a los padres. En segundo lugar, se procedió a la administración de las pruebas para identificar a los alumnos con altas habilidades (superdotados y talentos) participantes en este estudio. Finalmente, para la valoración de las competencias socioemocionales se utilizó el EQ-i:Yv. Las evaluaciones se hicieron dentro del horario lectivo.

\section{Análisis de datos}

Se llevaron a cabo diferentes análisis con el paquete estadístico spss versión i9 para Mac utilizando distintos procedimientos dentro de un método general de carácter descriptivo. Las técnicas de análisis fueron: a) análisis de frecuencias para comprobar la incidencia de alumnos talentosos; b) análisis descriptivos para el estudio de las dimensiones emocionales estudiadas, para los que utilizaron los coeficientes mínimo, máximo, media y desviación típica; c) análisis de diferencias de medias realizado mediante ANOva de un factor, para comprobar la existencia de diferencias en las distintas dimensiones de la inteligencia emocional.

\section{Resultados}

En la Tabla i se presentan los alumnos que se encuentran en cada uno de los perfiles intelectuales que se considerarán en el presente estudio.

Tabla I. Perfiles intelectuales de los alumnos identificados como talentos

\begin{tabular}{|c|c|l|}
\hline $\begin{array}{c}\text { FRECUENCIAS } \\
\text { DE LOS PERFILES } \\
\text { INTELECTUALES }\end{array}$ & \multicolumn{1}{|c|}{ CARACTERÍSTICAS } \\
\hline & & $\begin{array}{l}\text { La configuración intelectual que subyace en el talento figu- } \\
\text { rativo se fundamenta en la aptitud espacial, el razonamiento } \\
\text { lógico. Es propio de los individuos que manifiestan una gran } \\
\text { capacidad para percibir imágenes internas y externas, trans- } \\
\text { formarlas, modificarlas y descifrar la información gráfica. } \\
\text { No todos los alumnos que muestran capacidades visuales } \\
\text { exhiben las mismas habilidades. La evaluación de las distin- } \\
\text { tas aptitudes se debe realizar mediante diversas actividades } \\
\text { y escalas de tipo figurativo o espacial, de razonamiento ló- } \\
\text { gico. A la hora de valorar los productos, es necesario contar } \\
\text { además con las destrezas de ejecución o de utilización de los } \\
\text { instrumentos. }\end{array}$ \\
\hline \multirow{2}{*}{ I03 Talentos figurativos }
\end{tabular}




\begin{tabular}{|c|c|c|}
\hline & $\begin{array}{l}\text { FRECUENCIAS } \\
\text { DE LOS PERFILES } \\
\text { INTELECTUALES }\end{array}$ & CARACTERÍSTICAS \\
\hline \multirow{2}{*}{ 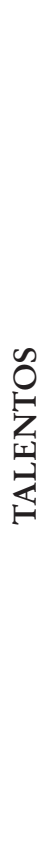 } & 34 Talentos académicos & $\begin{array}{l}\text { Es un tipo de talento complejo en el cual se combinan recur- } \\
\text { sos elevados de tipo verbal, lógico y de gestión de la memo- } \\
\text { ria. Los talentos académicos manifiestan una gran capacidad } \\
\text { para almacenar y recuperar cualquier tipo de información } \\
\text { que se pueda expresar verbalmente, suelen tener además una } \\
\text { buena organización lógica. Las funciones que manifiestan } \\
\text { los talentos académicos son idóneas para los aprendizajes } \\
\text { formales. Suelen trabajar bien con tareas verbales, numéri- } \\
\text { cas, espaciales, de memoria y de razonamiento, consideradas } \\
\text { todas ellas capacidades mentales primarias, necesarias para } \\
\text { lograr el éxito académico. }\end{array}$ \\
\hline & $\begin{array}{l}94 \text { Talentos combinados } \\
\text { (figurativos +académicos) }\end{array}$ & $\begin{array}{l}\text { Destacan por sus altas habilidades para entender y recordar } \\
\text { las relaciones espaciales entre objetos, facilidad para mani- } \\
\text { pular imágenes en el espacio, capacidad para visualizar, se- } \\
\text { parar y relacionar partes de un complejo sistema físico en el } \\
\text { espacio. Además, sus recursos verbales, lógicos, junto con } \\
\text { su extraordinaria memoria, les permiten tener una excelente } \\
\text { capacidad para aprender, resolver problemas y cuestiones } \\
\text { de tipo académico-práctico. También es extraordinaria su } \\
\text { capacidad para comprender los principios mecánicos y fí- } \\
\text { sicos en situaciones conocidas, para utilizarlos en ocupa- } \\
\text { ciones relacionadas con mecánica, mantenimiento de ma- } \\
\text { quinarias, etc. }\end{array}$ \\
\hline & AL 23I & \\
\hline
\end{tabular}

En la Tabla 2 se presentan los resultados obtenidos por los alumnos con talento (académico, figurativo o combinado) en las dimensiones de intrapersonal, interpersonal, estado de ánimo, manejo del estrés, adaptabilidad y Cociente Emocional. Se presenta la puntuación mínima y máxima, la media, la desviación típica, los análisis ANOva y los post-hoc.

En primer lugar, en cuanto a las medias, si comparamos a nivel descriptivo las puntuaciones obtenidas en inteligencia emocional por los alumnos, se observa que en las variables intrapersonal y adaptabilidad son los talentos combinados los que obtienen la mayor puntuación. En segundo lugar, los académicos son los que alcanzan mayores puntuaciones en la variable interpersonal, en el estado de ánimo y en el Cociente Emocional (Emotional Quotient). En tercer lugar, los talentos figurativos son los que obtienen mayores puntuaciones en manejo del estrés. La diferencia de medias en función de los perfiles cognitivos de alta habilidad (académico, figurativo y combinados) utilizados en este trabajo se pueden observar de forma visual en el Gráfico I. 
TABla 2. Estadísticos descriptivos de las puntuaciones de los alumnos según su configuración cognitiva en las dimensiones de la Inteligencia Emocional (IE), ANOVA y análisis post-hoc

\begin{tabular}{|c|c|c|c|c|c|c|c|}
\hline $\begin{array}{c}\text { Componentes } \\
\text { IE }\end{array}$ & Talentos & Media & DT & Mínimo & Máximo & ANOVA & $\begin{array}{l}\text { Post- } \\
\text { hoc }^{*}\end{array}$ \\
\hline \multirow[t]{3}{*}{ Intrapersonal } & Académicos & I4,I47 & 2,017 & 9 & 19 & $\mathrm{~F}(2,226)=4, \mathrm{I} \mathrm{II} ; \mathrm{p}=, \mathrm{OI} 7$ & $3>1$ \\
\hline & Figurativos & I3,44I & 2,947 & 6 & 20 & & \\
\hline & $\begin{array}{l}\text { Combinados } \\
\text { (Académico+Figurativo) }\end{array}$ & $\mathrm{I} 4,548$ & $2,63 \mathrm{I}$ & 7 & 24 & & \\
\hline \multirow[t]{3}{*}{ Interpersonal } & Académicos & 39,794 & 3,991 & 30 & 47 & $\mathrm{~F}(2,226)=0,572 ; \mathrm{p}=, 565$ & \\
\hline & Figurativos & $38,93 \mathrm{I}$ & 4,297 & 27 & 48 & & \\
\hline & $\begin{array}{l}\text { Combinados } \\
\text { (Académico+Figurativo) }\end{array}$ & 39,333 & 4,345 & 27 & 48 & & \\
\hline \multirow[t]{3}{*}{ Est. ánimo } & Académicos & 44,500 & 5,378 & 32 & 55 & $F(2,226)=0,033 ; p=, 968$ & \\
\hline & Figurativos & 44,265 & 6,092 & 28 & 56 & & \\
\hline & $\begin{array}{l}\text { Combinados } \\
\text { (Académico+Figurativo) }\end{array}$ & 44,452 & 5,988 & 28 & 56 & & \\
\hline \multirow[t]{3}{*}{ Manejo } & Académicos & 27,500 & 5,023 & I8 & 42 & $F(2,226)=3,068 ; p=, 048$ & $\mathrm{I}>3$ \\
\hline & Figurativos & $28,93 \mathrm{I}$ & 6,100 & 19 & 48 & & \\
\hline & $\begin{array}{l}\text { Combinados } \\
\text { (Académico+Figurativo) }\end{array}$ & 26,925 & 5,580 & 19 & 47 & & \\
\hline \multirow[t]{3}{*}{ Adaptabilidad } & Académicos & 29,735 & 4,187 & 22 & 40 & $\mathrm{~F}(2,226)=3,768 ; \mathrm{p}=, 025$ & $3>1$ \\
\hline & Figurativos & 29,333 & $4,6 \mathrm{I} 7$ & 16 & 40 & & \\
\hline & $\begin{array}{l}\text { Combinados } \\
\text { (Académico+Figurativo) }\end{array}$ & $3 \mathrm{I}, 075$ & 4,490 & 20 & 40 & & \\
\hline \multirow{3}{*}{ IE Total } & Académicos & I26,49I & 9,150 & 103,54 & I5I,6I & \multirow{3}{*}{$\mathrm{F}(2,226)=, 040 ; \mathrm{p}=, 96 \mathrm{I}$} & \\
\hline & Figurativos & I26,III & $\mathrm{II}, 583$ & 92,33 & 163,67 & & \\
\hline & $\begin{array}{l}\text { Combinados } \\
\text { (Académico+Figurativo) }\end{array}$ & $\mathrm{I} 25,833$ & 13,266 & 92,52 & 169,57 & & \\
\hline $\begin{array}{l}\text { * Nota. I. Tale } \\
\text { utilizado Ga }\end{array}$ & $\begin{array}{l}\text { ntos Figurativos; 2. Talento } \\
\text { mes-Howell }\end{array}$ & Acadén & $\cos ; 3 . \mathrm{T}$ & entos Co & mbinados & Figurativo + Académico)/ & ntraste \\
\hline
\end{tabular}


Gráfico I. Diferencia de medias en inteligencia emocional según los perfiles intelectuales

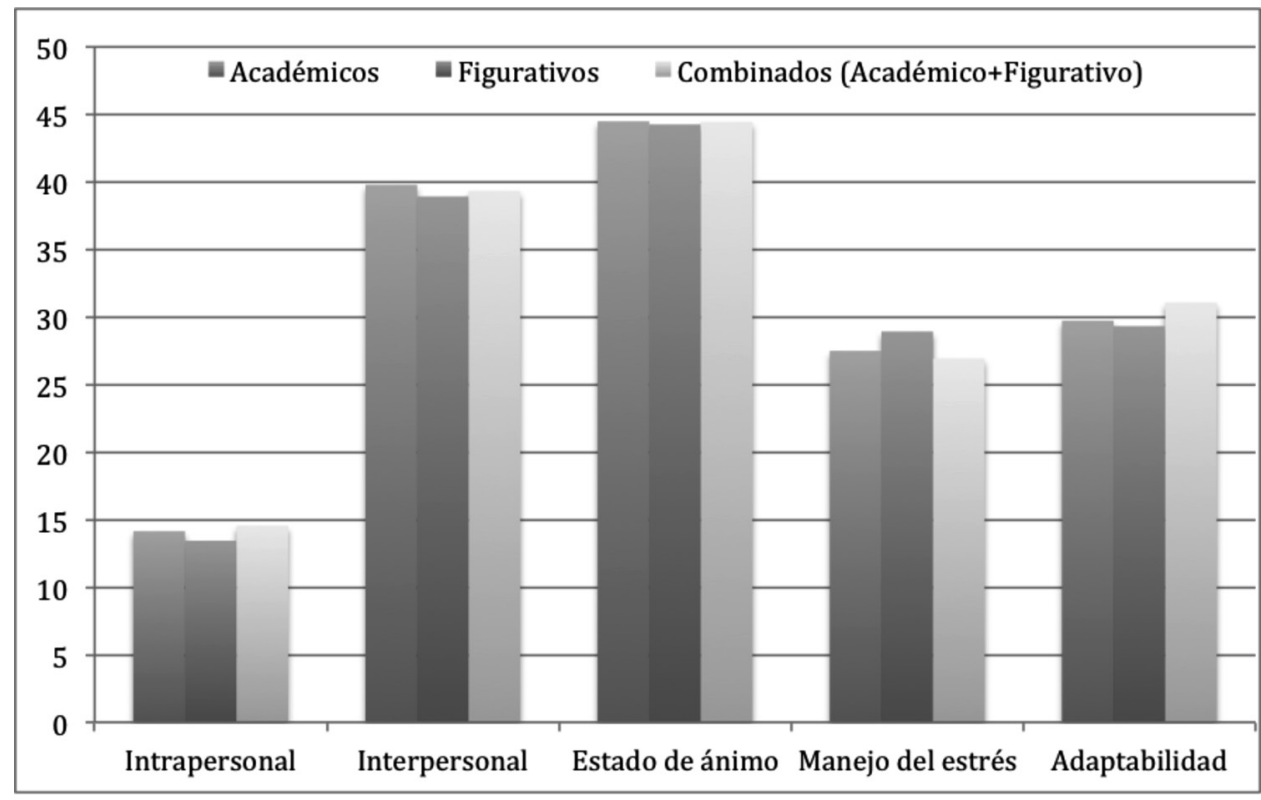

Con objeto de estudiar si las diferencias entre las puntuaciones medias observadas en inteligencia emocional entre los diferentes talentos eran estadísticamente significativas o se deben a mero error de muestreo aleatorio, se llevaron a cabo análisis de diferencias de medias. Los resultados mostraron diferencias estadísticamente significativas para el caso de las variables intrapersonal, manejo del estrés y adaptabilidad.

En la variable intrapersonal las diferencias fueron estadísticamente significativas entre los talentos combinados y los figurativos $(\mathrm{F}(2,226)=4, \mathrm{I} 5 \mathrm{I} ; \mathrm{p}=0, \mathrm{OI} 7)$, a favor de los talentos combinados, lo que significa que los talentos combinados muestran mayores habilidades de autoconciencia y de respeto personal. También se encontraron diferencias en adaptabilidad entre los talentos combinados y los figurativos $(\mathrm{F}(2,226)$ $=3,768 ; \mathrm{p}=0,025)$ a favor de los talentos combinados, lo que apunta a que éstos manifiestan una mayor flexibilidad para adaptarse a las diversas situaciones de la vida. Y, en la variable de manejo del estrés, las diferencias fueron estadísticamente significativas entre los talentos figurativos y los combinados $(\mathrm{F}(2,226)=3,068 ; \mathrm{p}=0,048)$ a favor de los talentos figurativos, es decir, los talentos figurativos manifiestan un mayor control de las situaciones estresantes (ver Tabla 2).

\section{Conclusiones y discusión}

A modo de conclusiones, podemos destacar que de los análisis se desprenden dos perfiles:

Uno, referido al talento figurativo (elevados recursos de representación y manipulación de variables; habilidad para establecer relaciones entre objetos y extraer reglas; 
habilidades espaciales para visualizar objetos en tres dimensiones), estudiantes que destacan por su capacidad de manejo del estrés. Esto significa que son estudiantes que bajo presiones académicas tienen una cierta capacidad para manejar y controlar sus emociones. Se puede decir que su capacidad les permite tolerar la presión y controlar sus impulsos. Estos datos concuerdan con otros estudios donde se destaca que el talento mecánico (capacidad para comprender principios básicos necesarios para resolver problemas, que exigen el manejo de mecanismos complejos) muestra grandes habilidades para controlar situaciones y circunstancias estresantes que exigen trabajar bajo presión (Sainz, Ruiz y Prieto, 20I4). En otros estudios se ha puesto de relieve que los talentos en su conjunto y los estudiantes con alta inteligencia manifestaron un mayor manejo del estrés que los superdotados (Prieto y Ferrando, 2009; Sainz, 20Io). Quizás, la interacción de diversas variables, propia de este tipo de talentos figurativos, hace que muestren habilidades adecuadas para soportar situaciones adversas, estresantes e incluso emociones fuertes, sin desmoronarse; pudiéndose enfrentar activa y positivamente al estrés, como destaca Bar-On (1997). Además, destaca que el manejo del estrés es el que mayor impacto tiene sobre las habilidades cognitivas; es decir, las habilidades cognitivas o inteligencia es la que está fuertemente influenciada por la habilidad del manejo de las emociones (Bar-On y Maree, 2009).

Otro es el talento combinado: académico y figurativo (recursos elevados de tipo verbal, lógico y de gestión de la memoria; además, elevados recursos de representación y manipulación de variables, lo que se traduce en una habilidad para establecer relaciones entre objetos y extraer reglas; habilidades espaciales para visualizar objetos en tres dimensiones). Este tipo de talento muestra elevadas competencias intrapersonales, junto con una alta adaptabilidad. De ello se desprende que son estudiantes con habilidades para el autoconocimiento emocional, asertividad, autoconsideración, autoactualización e independencia; es decir, son estudiantes que comprenden sus emociones, son capaces de expresarlas y saben la manera de comunicar sus sentimientos y necesidades. Estos datos coinciden con los resultados procedentes del trabajo de Schwean et al. (2006) y Fernández-Vidal (20II), quienes indican que los superdotados de su muestra se autopercibían con mayor inteligencia intrapersonal; sin embargo, Ferrando (2006) informó que los talentos como grupo puntuaban con menores destrezas intrapersonales que los superdotados. Bar-On encontró una relación entre la dimensión intrapersonal y la inteligencia psicométrica (Bar-On, 2004, 2007).

Este tipo de talento combinado también muestra una gran adaptabilidad o capacidad para tratar con los problemas cotidianos, resolverlos y tomar posibles decisiones para su resolución. Implica cierta flexibilidad para ajustarse a las emociones, los pensamientos y las conductas cuando cambian las situaciones y las condiciones. Estos datos sobre la adaptabilidad de este tipo de talentos coinciden con los hallados por otros autores, quienes encuentran diferencias entre la autopercepción de adaptabilidad que tienen los superdotados frente a los no superdotados (Schwean et al., 2006; Seon-Young y Olszewski-Kubilius, 2006). En el estudio de Ferrando (2006) los resultados indicaron que los alumnos de altas habilidades (superdotados y talentos) obtuvieron puntuaciones más elevadas que sus compañeros de habilidades medias en todas las áreas de la IE, siendo estas diferencias más significativas para la adaptabilidad; $y$ fueron los talentos quienes se autopercibieron con una mayor capacidad para la adaptabilidad que los superdotados. A resultados semejantes llegó Sainz (2010), quien indicó que el grupo de estudiantes de alta inteligencia se percibió con mayor adaptabilidad que sus compañeros con baja inteligencia. 
Esta mayor adaptabilidad apoya las posturas mantenidas por aquellos investigadores que indicaban que la capacidad cognitiva muy elevada implicaba un mayor ajuste psicológico. Los superdotados adolescentes desde muy pequeños afrontan las situaciones de estrés mejor, porque tienen un sentido más agudo sobre las diversas posibilidades de afrontamiento al estrés; o, dicho de otra manera, los superdotados están más abiertos y son más flexibles para afrontar los problemas (Burks, Jensen y Terman, I930; Cox, I926; Dirkes, I983; Hollingworth, I931, I942; Judá, I949; Lewis, I943; Schuler, 2002; Terman, I916, I925; Terman y Oden, 1947, I959; Zeidner y Schleyer, I999). Esta capacidad de afrontamiento al estrés y la flexibilidad se explican por la gran capacidad del superdotado para enfrentares mejor al estrés y a los conflictos, tal y como hemos recogido en los estudios analizados anteriormente (e. g. Baker, 1995; Galluci, 1988; Grossberg y Cornell, 1988; Howard, Hamilton y Franks, I995; Nail y Evans, I997; Neihart, I991; Olszewski-Kubilius y Kukieke, 1989).

Los datos resultantes de nuestro estudio sobre la adaptabilidad de los talentos contradicen las posturas mantenidas por aquellos expertos que sostienen que los superdotados y talentos tienen una mayor vulnerabilidad a desarrollar problemas de ajuste psicológico (Austin y Draper, 198r; Janos y Robinson, 1985; Lajoie y Shore, I98I; Powell y Haden, I984; Roedell, I984; Schauer, I976). Una de las razones que se argumenta es que su perfeccionismo les genera cotas altas de ansiedad y, por tanto, desajuste emocional (Pyryt, 20II).

Una de las cuestiones que nos ha sorprendido es que los talentos académicos con un razonamiento verbal alto, donde se combinan recursos elevados de tipo verbal, lógico y de gestión de la memoria, no hayan alcanzado mayores niveles en las competencias socioemocionales. Aunque esto es congruente con los datos hallados por algunos autores (Bermejo, Prieto, Fernández, Soto y Sainz, 2013), sin embargo, contradice los hallazgos que sostienen que la alta habilidad verbal puede contribuir modestamente al desarrollo de las competencias emocionales (Zeidner, Shani-Zinovich, Matthews y Roberts, 2005). Aunque como señalan estos autores este tipo de relaciones entre las habilidades cognitivas y emocionales requiere más investigación.

Para acabar queremos destacar que Bar-On y Maree (2009) indican que los superdotados son más habilidosos que sus compañeros en las siguientes dimensiones: manejo de sus emociones; asertividad para expresar sus sentimientos y mantener sus ideas; para validar objetivamente sus propias emociones; flexibilidad para adaptarse y ajustar sus emociones y pensamientos a situaciones nuevas; solución eficiente de problemas personales e interpersonales; optimismo para mantener una actitud positiva ante la vida, mirando siempre el lado más positivo de la vida; y autorrealización referida a la capacidad para esforzarse para alcanzar las metas de logro y actualizar su potencial. Sin embargo, hay que ser cautelosos con esta afirmación, porque hemos de seguir haciendo más trabajos donde se incorporen diferentes medidas de la capacidad cognitiva, así como de las competencias socioemocionales, como puede ser el modelo de habilidades de la inteligencia emocional. Ésta es una limitación de nuestro estudio. 


\section{Bibliografía}

Austin, A. B. y Draper. D. C. (198I) Peer relationships of the academically gifted: A review. Gifted Child Quarterly, 25, I29-133. http://dx.doi.org/I0.II77/001698628102500310

Baker, J. A. (1995) Depression and suicidal ideation among academically gifted adolescents. Gifted Child Quarterly, 39, 218-223. http://dx.doi.org/Io.1177/001698629503900405

BAR-On, R. (1997) The Bar-On Emotional Quotient Inventory (EQ-i): A Test of Emotional Intelligence. Toronto, Canada: Multi-Health Systems.

BAR-On, R. (2OOI) Emotional intelligence and self-actualization. En Joseph Ciarrochi, Joe FORGAS y John D. MAYER (eds.) Emotional intelligence in everyday life: A scientific inquiry (pp. 82-97). New York: Psychology Press.

BAR-ON, R. (2004) The Bar-On Emotional Quotient Inventory (EQ-i): Rationale, description, and summary of psychometric properties. En G. GeHER (ed.) Measuring emotional intelligence: Common ground and controversy (pp. III-I42). Hauppauge, NY: Nova Science Publisher.

BAR-ON, R. (2006) EQ-i leadership user's guide. Toronto, Canada: Multi-Health Systems.

BAR-On, R. (2007) The Bar-On model of emotional intelligence: A valid, robust and applicable EI model. Organisations and People, I4, 27-34.

BAR-ON, R. y MAREE, J. G. (2009) In search of emotional-social giftedness: A potentially viable and valuable concept. En L. V. SHAvinina (ed.) International handbook of giftedness (pp. 559-570). New York City: Springer Science. http://dx.doi.org/I0.1007/978-I-4020-6162-2_26

BAR-On, R. y PARker, J. D. (2000) Bar-On Emotional Quotient Inventory: Youth VersionObserver Form. Toronto, Canada: Multi-Health Systems.

Bermejo, R.; Prieto, M. D.; Fernández-Vidal M. C.; Soto, G. y Sainz, M. (20I3) Perfil Cognitivo-Creativo del Talento Emocional. Journal of New Approaches In Educational Research (NAER), 2 (I), I3-I7. http://dx.doi.org/I0.782I/naer.2.I.I2-16

Burks, B. S.; Jensen, D. W. y Terman, L. (1930) Genetic studies of genius: Volume 3, The promise of youth: Follow-up studies of a thousand gifted children. Stanford, CA: Stanford University Press.

Castelló, A. y Batlle, C. (1998) Aspectos teóricos e instrumentales en la identificación del alumnado superdotado y talentoso. Propuesta de un protocolo. FAISCA, 6, 26-66.

Cox, C. M. (1926) The early mental traits of three hundred geninses. Stanford, CA: Stanford University Press.

Dirkes, M. A. (1983) Anxiety in the gifted: Pluses and minuses. Roeper Review, 6, 68-60. http://dx.doi.org/Io.I080/02783198309552758

FERnández-VIdAL, M. C. (2OII) Competencia socioemocional en adolescentes de altas habilidades: Un estudio comparativo. Tesis doctoral. Universidad de Murcia.

Ferrando, M. (2006) Creatividad e Inteligencia Emocional: Un estudio empírico en alumnos con altas habilidades. Tesis doctoral. Universidad de Murcia.

Gallucci, N. T. (1988) Emotional adjustment of gifted children. Gifted Child Quarterly, 32, 273-276.

http://dx.doi.org/Io.II77/oor698628803200206

Gross, J. J. (20I4) Emotion regulation: conceptual and empirical foundationes. En Handbook of Emotion Regulation (pp. 3-20). New York: The Guildford Press.

Grossberg, I. N. y Cornell, D. G. (1988) Relationship between personality adjustment and high intelligence: Terman versus Hollingworth. Exceptional Children, 55, 266-272.

HollingwORTH, L. S. (I93I) The child of very superior intelligence as a special problem in social adjustment. Mental Hygiene, I5, I-6I. 
Hollingworth, L. S. (1942) Children above 180 IQ Stanford-Binet: Origin and development. Yonkers-on-Hudson, NY: World Book. http://dx.doi.org/IO.IO37/13574-000

Howard-Hamilton, M. y Franks, B. A. (1995) Gifted adolescents: Psychological behaviors, values, and developmental implications. Roeper Review, I7(3), I86-19I.

http://dx.doi.org/IO.IO80/02783199509553656

IBм Corp. (20II) IBM SPSS Statistics for Windows, Version 20.0. Armonk, NY: IвM Corp.

Janos, P. M. y Robinson, N. M. (1985) Psychosocial development in intellectually gifted children. En F. D. Horowitz y M. O'Brien (eds.) The gifted and talented: Developmental perspectives. Washington, DC: American Psychological Association. http://dx.doi.org/I0.1037/I0054-006

JudA, A. (1949) The relationship between highest mental capacity and psychic abnormalities. American Journal of Psychiatry, 106, 296-307. http://dx.doi.org/Io.II76/ajp.I06.4.296

Kane, M. y Silverman, L. (20I4) Fostering Well-Being in Gifted Children: Preparing for an Uncertain Future. En F. Hellen, M. Machado, S. Bahia y T. Stoltz. Altas Habilidades /Superdotação (AH/SD). Criatividade e Emoção (pp. 67-83). Brasil: Juruá.

Lajoie, S. P. y Shore, M. (I98I) Three myths? The over-representation of the gifted among dropouts, delinquents, and suicides. Gifted Child Quarterly, 25, I38-I43. http://dx.doi.org/Io.II77/001698628102500312

LEWIS, W. D. (1943) Some characteristics of very superior children. Journal of Genetic, 62, 30I309.

http://dx.doi.org/ı0.1080/08856559.1943.10533220

Nail, J. M. y Evans, J. G. (1997) The emotional adjustment of gifted adolescents: A view of global functioning. Roeper Review, 20, I8-2I. http://dx.doi.org/Io.IO80/02783199709553845

NeIHART, M. (I99I) Anxiety and depression in high ability and average ability adolescents. Doctoral dissertation. University of Northern Colorado. Dissertation Abstracts International.

Neville, C.; Tolan, S. S. y Piechowski, M. M. (eds.) (2013) Off the charts. Asynchrony and the gifted child. Unionville, NY: Royal Fireworks Press.

Olszewski-Kubilius, P. y Kulieke, M. (1989) Personality dimensions of gifted adolescents. En J. VanTassel-Baska \& P. Olszewski-Kubilius (eds.) Patterns of Influence on Gifted Learners: the Home, the Self, and the School (pp. 125-145). New York: Teachers College Press.

Peterson, J. (20I4) Paying attention to the Whole Gifted Child: Why, When, and How to focus on Social and Emotional Development. En F. Hellen, M. Machado, S. Bahia y T. Stoltz. Altas Habilidades/Superdotação (AH/SD). Criatividade e Emoção (pp. 45-65). Brasil: Juruá.

Powell, P. M. y Haden, T. (1984) The intellectual and psychosocial nature of extreme giftedness. Roeper Review, 6, I3I-I33. http://dx.doi.org/Io.IO80/02783198409552783

Prieto, M. D. y Ferrando, M. (2009) Prejudices about Emotional Intelligence in Gifted and Talented Children. En T. Balchin, B. Hymer y D. Matthews (eds.) The Routledge International Com-panion to Gifted Education (pp. I49-154). New York: Routledge-Farmer.

Pyryt, M. C. (2OII) Helping gifted students cope with perfectionism. En J. L. Jolly, D. J. TRefFinger, T. F. Inman y J. F. Smutny (eds.) Parenting gifted children (pp. 546-554). Waco, TX: Prufrock Press.

Roedell, W. C. (1984) Vulnerabilities of highly gifted children. Roeper Review, 6, I27- I30. http://dx.doi.org/I0.1080/02783198409552782

SAInz, M. (2008) Competencia socioemocional de alumnos con altas habilidades y habilidades medias: Percepción de padres, profesores y alumnos. Tesis de Licenciatura. Universidad de Murcia. 
SAInz, M. (20Io) Creatividad, personalidad y competencia socio-emocional en alumnos de Altas Habilidades versus no Altas Habilidades. Tesis doctoral. Universidad de Murcia.

Sainz, M.; Fernández-Vidal M. C. y Soto, G. (20I2) Inteligencia Emocional y Alta Habilidad. Revista Amazônica, x, 186-203.

Sainz, M.; Ferrándiz, C.; Fernández, M. C. y Ferrando, M. (20I4) Propiedades psicométricas del Inventario de Cociente Emocional EQ-i:Yv en alumnos superdotados y talentosos. Revista de Investigación Educativa, 32 (I), 4I-55. http://dx.doi.org/I0.60I8/rie.32.I.I6250I

Sainz, M.; Ruiz, M. J. y Prieto, L. (20I4) Perfiles emocionales de superdotados y talentos. En F. H. Ribeiro, M. Machado, S. Bahia y T. Stoltz (coords.) Altas Habilidades y Superdotação (AH/SD). Criatividades e Emoção (pp. I27-I48). Brasil: Juruá.

Schauer, G. H. (1976) Emotional disturbance and giftedness. The Gifted Child Quarterly, 20 (4), $470-477$.

Schewean, V. L.; Saklofske, D. H.; Widdifield-Konkin, L.; Parker, J. y Kloosterman, P. (2006) Emotional Intelligence and Gifted Children. E-Journal of Applied Psychology: Emotional Intelligence, 2 (2), 30-37. http://dx.doi.org/Io.7790/ejap.v2i2.70

Schuler, P. (2002) Perfectionism in gifted children and adolescents. En M. Neihart, S. M. Reis, N. M. Robinson y S. M. Moon (eds.) The social and emotional development of gifted children: What do we know? (pp. 7I-79). Waco, Tx: Prufrock Press.

Seon-Young, L. y Olszewski-Kubilius, P. (2006) The Emotional Intelligence, Moral Judgment and Leadership of Academically Gifted Adolescents. Journal for the Education of the gifted, 30 (I), 29-67.

SwORD, L. K. (2005) Emotional intensity in gifted children. Obtenido de www.giftedservices. com.au.

Terman, L. (1916) The measurement of intelligence. Boston: Houghton Mifflin. http://dx.doi.org/10.1037/I0oI4-ooo

Terman, L. (1925) Mental and physical traits of a thousand gifted children. Genetic studies of genius, vols. I and 2. Stanford, CA: Stanford University Press.

Terman, L. y Oden, M. H. (1947) The gifted child grows up. Genetic studies of genius, vol. 4 . Stanford, CA: Stanford University Press.

Terman, L. y Oden, M. H. (1959) The gifted group at mid-life. Genetic studies of genius, vol. 5. Stanford, Ca: Stanford University Press.

Zeidner, M. y SchleYer, E. J. (1999) The big-fish-little-pond effect for academic self concept, test anxiety, and school grades in gifted children. Contemporary Educational Psychology, $24,305-329$. http://dx.doi.org/IO.IOo6/ceps.1998.0985

Zeidner, M.; Shani-Zinovich, I.; Matthews, G. y Roberts, R. D. (2005) Assessing Emotional Intelligence in gifted and non-gifted school students: Outcomes depending on the measure. Intelligence, 33, 369-391.

http://dx.doi.org/I0.1016/j.intell.2005.03.00I

\section{Agradecimientos}

Este trabajo se ha realizado con diferentes ayudas: a) Fundación Séneca-Agencia de Ciencia y Tecnología de la Región de Murcia (Ref.: ıı896/PHCS/o9). b) Ministerio de Ciencia y Tecnología (EDU2010-16370). 